\title{
Discharge-Driven 46.9-nm Amplifier with Gain-Length Approaching Saturation
}

\author{
J. J. Rocca, Senior Member, M. C. Marconi, J. L. A. Chilla, D. P. Clark, F. G. Tomasel, and V. N. Shlyaptsev
}

\begin{abstract}
Gain length products up to $g l \approx 14$ for the $J=0-1$ line of Ne-like Ar at $46.9 \mathrm{~nm}$ have been achieved in $15-\mathrm{cm}-$ long plasma columns generated by a fast capillary discharge. Amplification in plasma columns up to $20 \mathrm{~cm}$ in length was investigated. The laser line intensity is observed to increase exponentially for plasma lengths of up to $15 \mathrm{~cm}$, above which it is observed to saturate. The saturation behavior is discussed.
\end{abstract}

\section{INTRODUCTION}

$\mathbf{T}$ HE FIRST demonstrations of large soft X-ray amplification in laser created plasmas, achieved utilizing two different excitation mechanisms, collisional excitation and recombination, yielded similar gain length products in the range of 6-7 [1], [2]. Since then, significant progress has been made in increasing the intensity of laser pumped soft $\mathrm{X}$-ray amplifiers [3]-[11]. In cases in which limiting effects, such as refraction, can be overcome, the amplified spontaneous emission intensity increases exponentially as a function of plasma column length until saturation of the gain is reached. Several experiments with laser-pumped collisionally excited lasers have succeeded in demonstrating saturated or nearsaturated operation [3]-[10]. In collisionally excited lasers in Ne-like ions gain saturation, which is dependent on the geometry of the amplifier, has been typically achieved at $g l$ values between 14 and 20 . Gain saturation was observed to occur at $g l \approx 14$ in Ne-like Ge [5], [6] and Ne-like Zn [8] and at $g l \approx 15-17$ in Ne-like Se [4].

We recently reported the first demonstration of large soft $\mathrm{X}$-ray amplification in a plasma column generated by an electrical discharge [12]. This new excitation scheme has potential for increasing the simplicity and wall-plug efficiency of ultrashort-wavelength lasers. In the initial experiments a gain-length product of 7.2 was obtained at $46.9 \mathrm{~nm}$ by collisionally exciting the $J=0-1$ line of Ne-like Ar in a 12-cm-long capillary discharge plasma. Herein we report operation of a discharge-pumped $46.9 \mathrm{~nm}$ amplifier at gain-

Manuscript received June 5, 1995, revised July 5, 1995. This work was supported in part by the National Science Foundation under grants ECS9401952 and ECS-9412106, and the U.S. National Research Council. Part of the diagnostics instrumentation used in this work was developed in collaboration with Hyperfine Inc, (Boulder, $\mathrm{CO}$ ) with the support of the Colorado Advanced Technology Institute.

J. J. Rocca, J. L. A. Chilla, D. P. Clark, and F. G. Tomasel are with the Department of Electrical Engineering, Colorado State University, Fort Collins, CO 80523 USA.

M. C. Marconi is with the Departamento de Física, Universidad de Buenos Aires, Buenos Aires, Argentina.

V. N. Shlyaptsev is with the P. N. Levedev Physical Institute, Moscow, Russia.

IEEE Log Number 9414563 length products of up to $g l \approx 14$, corresponding to a laser intensity more than two orders of magnitude greater than previously reported [12]. The increased gain-length product, which approaches the values necessary for gain saturation of the laser line, is the result of an increased gain coefficient resulting from more favorable discharge conditions, and a larger plasma column length. The experiments were conducted producing discharges up to $20 \mathrm{~cm}$ in length through 4-mmdiameter polyacetal capillaries. The fast discharge current pulse rapidly compresses and heats the plasma to form a narrow column [13], which in the experiments discussed herein reaches aspect ratios exceeding 600:1. Toward the final stage of the compression the plasma reaches nearly ideal conditions for soft X-ray amplification by collisional excitation in Ne-like and Ni-like ions [13].

\section{AMPLIFICATION MEASUREMENTS}

The pulse generator and capillary discharge set-up used to conduct the experiments were described in a previous publication [14]. It consists of a 3-nF liquid dielectric capacitor that is pulsed charged by a Marx generator and is rapidly discharged through the capillary channel by a spark gap pressurized with $\mathrm{SF}_{6}$. To conduct the experiments discussed herein, the original discharge set-up was modified to allow for the use of capillary loads up to $20 \mathrm{~cm}$ in length. The axial spectrum emanating from the hollowed ground discharge electrode was recorded with a $2.2-\mathrm{m}$ grazing incidence spectrograph having a 1200 lines $/ \mathrm{mm}$ gold-coated grating placed at $85.8^{\circ}$. The radiation from the capillary plasma was directed onto the spectrometer slit by utilizing a mirror placed at grazing incidence. A gated micro-channelplate intensified-charge-coupled device array detector was used to detect the soft X-ray radiation. A flat-top 30-ns gate pulse centered at a time near the peak of the current pulse was applied to the detector to record the laser radiation. Variable attenuation of the laser intensity up to $4 \times 10^{3}$ was obtained by combining aluminum filters of various thicknesses. The filter transmissivities were calibrated utilizing the emission from the $46.07 \mathrm{~nm}$ and $46.24 \mathrm{~nm}$ lines of NeII produced by a CW low-pressure capillary discharge lamp.

All the measurements discussed herein were conducted in capillaries filled with pure argon. The variation of the $46.9 \mathrm{~nm} \mathrm{Ne}$-like Ar laser intensity as a function of plasma column length was measured for capillary channels of various lengths ranging from 3 to $20 \mathrm{~cm}$. The amplitude and period of the current were maintained approximately constant for all capillary discharge lengths at $39 \pm 1.5 \mathrm{kA}$ and $75 \pm 3 \mathrm{~ns}$, 


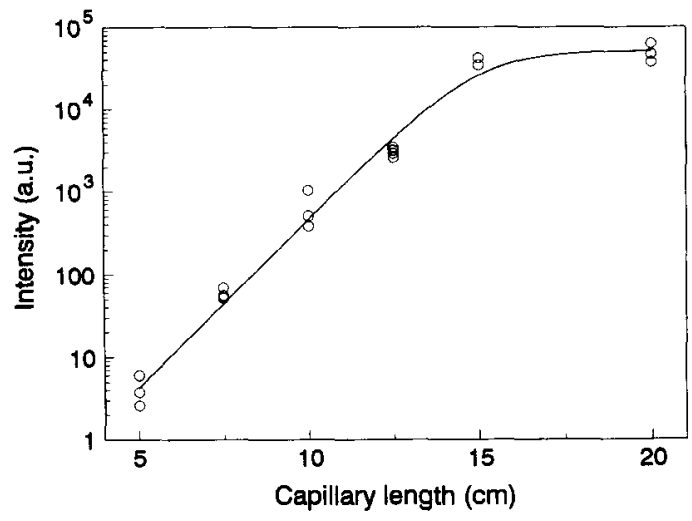

Fig. 1. Integrated line intensity of the 46.9-nm line of ArIX as a function of plasma column length. The capillary diameter is $4 \mathrm{~mm}$ and the argon pressure is $\approx 720$ mtorr. The maximum $g l$ value reached is 14.1 or 13.4 , depending on two slightly different measurements of the attenuation of the filters used. The corresponding gain coefficient for lengths $<15 \mathrm{~cm}$ determined by the Linford formula [15] are $0.94 \mathrm{~cm}^{-1}$ and $0.89 \mathrm{~cm}^{-1}$, respectively.

respectively. This was achieved by adjusting the discharge voltage for each capillary length and by adding inductance to the discharge circuit of the shorter capillaries to match that of the 20-cm-long channels. Fig. 1 shows the variation of the integrated line intensity of the 46.9-nm laser line as a function of capillary length for discharges at an argon pressure of about 720 mtorr. An increase of the plasma column length from 5 to $15 \mathrm{~cm}$ was measured to increase the intensity of the laser line by nearly four orders of magnitude. A fit of the data with the Linford formula [15] shows that the exponential increase of the intensity observed for plasma column lengths up to $15 \mathrm{~cm}$ corresponds to a gain coefficient of $0.94 \mathrm{~cm}^{-1}$ or $0.89 \mathrm{~cm}^{-1}$, depending on the use of two slightly different filter calibrations resulting from measurements conducted either before or immediately after the set of laser intensity measurements respectively. It should be noticed that the measured gain coefficient is the effective gain, $g_{\text {eff }}$, having a reduced value [16] with respect to the maximum gain coefficient $g_{0}=\sigma \Delta N$ which would occur in the absence of refraction. At a plasma length of about $15 \mathrm{~cm}$, when $g l$ reaches a value between 13 and 14 , the intensity increase is observed to saturate. The maximum $g l$ value reached is 14.1 or 13.4 depending on the calibration of the filter attenuation used.

Fig. 2 shows a set of laser line intensity measurements as a function of capillary length corresponding to discharges at an argon pressure of about $620 \mathrm{mtorr}$, excited by the same current pulse. The exponential increase was measured to correspond to a slightly larger effective gain coefficient of $1.09 \mathrm{~cm}^{-1}$, and the saturation behavior is observed at a shorter plasma length, around $12 \mathrm{~cm}$. While in this case of higher gain coefficient the roll-over of the intensity occurs at a shorter plasma length, as can be expected from gain saturation, the maximum $g l$ is lower however, reaching 12.8 .

The variation of the intensity of the $46.9 \mathrm{~nm}$ amplified spontaneous emission (ASE) with plasma length was computed using expressions developed by Casperson [17] for an inhomogeneously broadened line. Assuming a column having a gain region of $300 \mu \mathrm{m}$ diameter and a laser upper level radiative-to-effective lifetime ratio of 20 , determined from

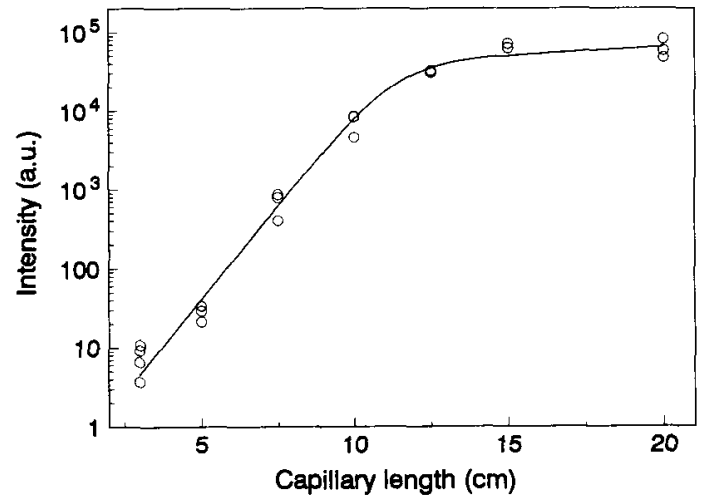

Fig. 2. Integrated line intensity of the $46.9-\mathrm{nm}$ line of ArIX as a function of plasma column length for an argon pressure of $\approx 620$ mtorr. The gain coefficient determined by a fit of the data for lengths $<10 \mathrm{~cm}$ to the Linford formula is $1.09 \mathrm{~cm}^{-1}$.

our atomic calculations, the calculation indicates that gain saturation should be reached at $g l \approx 18-19$. It should be noted that this calculation does not include refraction effects. The presence of refraction produces a concentration of the most intense rays that, for some geometries, can be shown to reduce the value of $g_{\text {eff }} l$ product at saturation [18]. It is possible, however, that an effect other than gain saturation is limiting the maximum amplification length. The role of possible limiting effects is discussed below.

\section{Beam Profile AND Refractive EFFects}

Refractive effects due to density gradients that can steer the laser beam out from the gain region could, if sufficiently strong, cause a roll-over of the amplification [16]. To gain understanding of the role of refraction in the observed amplification behavior, we conducted measurements of the angular intensity distribution of the laser-beam pattern. The beam pattern of an amplifier with cylindrical geometry can display a central lobe, corresponding to rays that propagate near the axis of the plasma column, surrounded by a concentric ring due to rays that are refracted off the gain region, as schematically illustrated in Fig. 3. When observed through a spectrograph, the corresponding image presents two side lobes if refraction is important. The toroidal mirror previously used between the plasma and the spectrometer [12] mixes spatial and angular information and for these measurements was substituted by either a planar or a cylindrical mirror with a $13-\mathrm{cm}$ radius of curvature in the direction parallel to the slit. The small source size combined with the relatively long distance between the plasma and the detector (approximately $2 \mathrm{~m}$ ), allows for an accurate determination of the angular distribution of the radiation intensity. However, with the planar mirror the maximum observed angle along the direction of the slit was limited to a maximum of about $7 \mathrm{mrad}$ by the length of the slit and the aperture of the aluminum filters utilized to attenuate the laser intensity. To be able to record laser radiation that might be emitted at larger angles, the beam was collimated to form an approximately parallel beam by placing the cylindrical mirror at mid-distance between the plasma and the spectrometer. In this case, in which the beam collimation after the grazing 


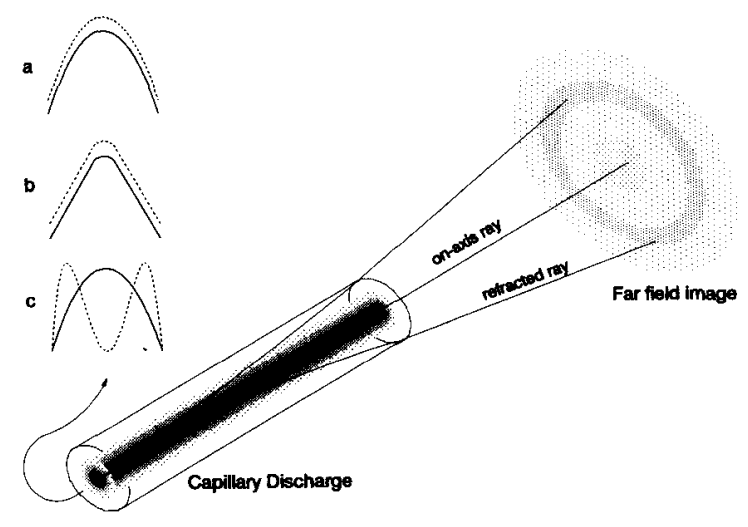

Fig. 3. Schematic representation of the effect of refraction in a plasma of cylindrical geometry. The inset shows the different spatial profiles of electron density (solid line) and gain (dashed lines) discussed in the text.

incidence curved mirror depends strongly on the angle of incidence, the calibration in angle was obtained by performing several laser shots in the presence of a fiducial array of slits.

Within the discharge parameter range for large amplification the beam pattern is often observed to present two off-axis lobes that, as discussed below, are a consequence of refraction. Fig. 4(a) shows the beam patterns at the detector plane of the spectrograph for capillary length of $7.5,15$, and $20 \mathrm{~cm}$ at the discharge conditions of Fig. 2. The data correspond to a series of shots in which the side lobes are most prominent. Other series of shots in different capillaries have also shown beam profiles with less prominent side lobes, such as that shown in Fig. 4(b). As shown by London [16], the angular separation of the side-lobes is determined only by the value of the electron density. The angular separation of the two off-axis side lobes in the measured beam patterns of Fig. $4(\mathrm{a})$ is $\approx 5.7 \mathrm{mrad}$ for the 10 - and $15-\mathrm{cm}$-long plasma columns. This divergence corresponds to an electron density for the gain region of $4 \times 10^{18} \mathrm{~cm}^{-3}$. This density is in good agreement with that predicted for the gain region by hydrodynamic/atomic physics calculations.

The possible role of refraction in limiting the amplification before gain saturation is achieved requires careful analysis as it is highly dependent on the geometry of the plasma and on the shape of the density and gain profiles. London [16] has analyzed the X-ray laser beam propagation in an exploding foil amplifier geometry with a one dimensional parabolic density profile. That analysis shows that the length dependence of the laser intensity corresponds to one of two cases determined by whether the refraction gain length $G_{r}=g_{0} L_{r}$ is smaller or larger than 1 . Here $g_{0}$ is the gain on axis and $L_{r}$ is the characteristic distance traveled by a ray within the lasing medium. When refraction limits the amplification $\left(G_{r}<1\right)$, the laser intensity as a function of plasma length rolls over and reaches a constant value before gain saturation is reached.

For the capillary discharge amplifier the essentials of that analysis are still valid, but it must be modified to take into account the two orthogonal components of the density gradient and to allow for the possibility a of nonparabolic density profile. The major difference introduced by the cylindrical geometry is that exponential growth is maintained if $G_{r}>2$.
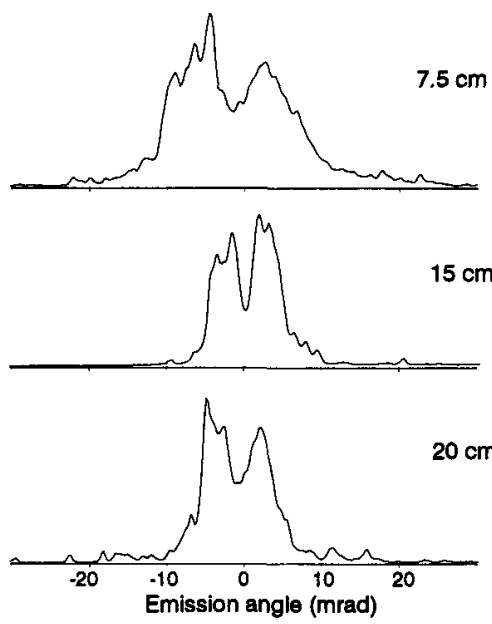

(a)

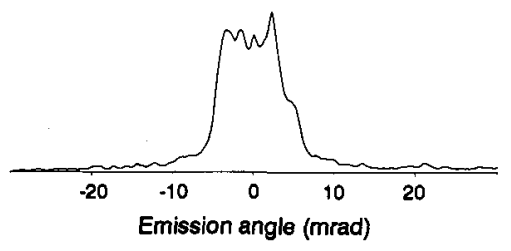

(b)

Fig. 4. (a) Beam patterns from 46.9-nm laser beams produced by capillary discharge plasma columns $7.5,15$, and $20 \mathrm{~cm}$ in length. Different combination of $\mathrm{Al}$ filters were used in each case to attenuate the laser beam and avoid saturation of the detector. The capillary diameter is $4 \mathrm{~mm}$ and the argon pressure is $\approx 620$ mtorr. The peak discharge current is $39 \mathrm{kA}$. (b) $46.9-\mathrm{nm}$ laser beam pattern with less-prominent side lobes.

It can also be shown that for arbitrary density profiles, the role of refraction in limiting amplification is determined by the curvature of the index of refraction profile in the vicinity of the maximum density, and that the refraction gain length can be defined as $G_{r}=g\left(r_{\max }\right) / \sqrt{\partial^{2} \mu / \partial r^{2}}$ where $g\left(r_{\max }\right)$ and $\partial^{2} \mu / \partial r^{2}$ are, respectively, the gain and second derivative of the index of refraction at the radial position of maximum density [18]. The high sensitivity of the role of refraction on the shape of gain and density profiles is evident from the definition of $G_{r}$. If density and gain profiles in the capillary plasma are assumed to a good approximation to be parabolic [Fig. 3(a)], refraction cannot be responsible for the observed saturation. In this case, the condition $G_{r}<2$ for the measured value of $g_{0} \geq 1 \mathrm{~cm}^{-1}$ would require $L_{r}<2 \mathrm{~cm}$, which is incompatible with the observed exponential growth of the laser intensity for lengths of up to $15 \mathrm{~cm}$. However, it is possible according to hydrodynamic/atomic physics calculations for the plasma column to have a narrowly peaked density profile [Fig. 3(b), large $\partial^{2} \mu / \partial r^{2}$ ], resulting in a small $G_{r}$ yielding both a dependence of laser power on plasma column length and beam profiles with dominant side lobes compatible with those observed in the experiments. The observed features could also result from a different configuration having small $G_{r}$, a parabolic density profile with annular gain [Fig. 3(c), small $g\left(r_{\max }\right)$ ]. This case is, however, less likely in our experiment, as measurements indicate that maximum amplification occurs very near the axis of the discharge [13]. 
Another possible cause of the observed roll-over of the exponential growth is the variation of the the gain in each given radial position, which can limit the effective amplification length. Spatially integrated streak camera measurements of the laser pulse emitted by a 12 -cm-long plasma column show that the laser pulse duration is $\approx 1 \mathrm{~ns}$, which should allow in the absence of gain saturation amplification in plasma columns up to $30 \mathrm{~cm}$ in length. However, due to the high radial velocity of the soft X-ray emitting region of the plasma during the final stage of the compression, approximately $2 \times 10^{7} \mathrm{~cm} / \mathrm{s}$ [13], the gain duration at each radial position might be shorter, thereby limiting the maximum effective amplification length. Further studies are necessary to clearly identify the cause of the observed saturation. In any case, further increase of the gainlength product might be achieved in double-pass amplification with the use of a spherical mirror to re-direct the beam back over its path toward the central region of the plasma column.

\section{SUMMARY}

In summary, gain-length products up to $\approx 14$ and gain coefficients larger than $1 \mathrm{~cm}^{-1}$ have been obtained in the $J=0-1$ line of Ne-like Ar in a capillary discharge plasma. The maximum $g l$ product was obtained in $15-\mathrm{cm}$-long plasma columns, the length above which the intensity is observed to saturate. This is, to our knowledge, the largest amplification reported from a table-top XUV amplifier and the first time a discharge-pumped amplifier is operated with a gain-length product approaching saturation.

\section{ACKNOWLEDGMENT}

The authors thank J. J. Gonzalez for his contributions to the experiments and O. E. Martinez for useful discussions.

\section{REFERENCES}

[1] D. L. Matthews, P. L. Hagelstein, M. D. Rosen, M. J. Eckart, N. M. Ceglio, A. U. Hazi, M. Medecki, B. J. Mac Gowan, J. E. Trebes, B. L. Whitten, E. M. Campbell, C. W. Hatcher, A. M. Hawryluk, R. L. Kauffman, L. D. Pleasance, G. Rambach, J. H. Scofield, G. Stone, and T. A. Weaver, "Demonstration of a soft X-ray amplifier," Phys. Rev. Lett., vol. 54, p. 110, 1985.

[2] S. Suckewer, C. H. Skinner, H. Milchberg, C. Keane, and D. Vorhees, "Amplification of stimulated soft X-ray emission in a confined plasma column," Phys. Rev. Lett., vol. 55, p. 1753, 1985.

[3] D. Matthews, M. Rosen, S. Brown, N. Ceglio, D. Eder, A. Hawryluk C. Keane, R. London, B. MacGowan, S. Maxon, D. Nilsen, J. Scofield and J. Trebes, "X-ray laser research at the Lawrence Livermore National Laboratory Nova Laser facility," J. Opt. Soc. Am., vol. 4, p. 575, 1987.

[4] B. J. MacGowan, L. B. DaSilva, and D. J. Fields, "Short wavelength X-ray laser research at the Lawrence Livermore National Laboratory Nova Laser facility," Phys. Fluids B, vol. 4, p. 2326, 1992.

[5] A. Carillon, H. Z. Chen, P. Dhez, L. Dwivedi, J. Jacoby, P. Jaegle, G. Jamelot, J. Zhang, M. H. Key, A. Kidd, A. Klishnick, R. Kodama, J. Krishnan, C. L. S. Lewis, D. Neely, P. Norreys, D. O'Neill, G. J. Pert S. A. Ramsden, J. P. Raucourt, G. J. Tallents, and J. Uhomoibhi, "Saturated and near-diffraction limited operation of an XUV laser at 23. 6 nm," Phys. Rev. Lett., vol. 68, p. 2917, 1992.

[6] S. Wang, G. Zhou, G. Zhang, J. Zheng, and S. Chunyu, "Overview of research on Ne-like Ge soft-X-ray laser in China," in AIP Conf. Proc. 332, X-ray Lasers 1994, Fourth Int. Colloquium, Williamsburg, VA, D. Eden and D. Matthews, Eds., pp. 293-300, 1994.
[7] C. L. Lewis, D. Neely, D. M. O'Neil, J. Ouhomoibhi, M. H. Key, Y. Al Hadithi, G. J. Tallents, and S. A. Ramsden, "An injection/amplifier double target configuration for the Ne-like $\mathrm{Ge} \mathrm{X}$-ray laser scheme," Opt. Comm., vol. 91, p. 71, 1992.

[8] B. Rus, A. Carillon, P. Dhez, B. Gauthé, P. Goedtkindt, P. Jaeglé, G. Jamelot, A. Klisnick, M. Nantel, A. Sureau, and P. Zeitoun, "Experimental study of Ne-like zinc $j=0-1$ soft X-ray lasing at $21.2 \mathrm{~nm}$," in AIP Conf. Proc. 332, X-ray Lasers 1994, Fourth Int. Colloquium, Williamsburg, VA, D. Eden and D. Matthews, Eds., pp. 152-156, 1994.

[9] L. B. Da Silva, B. J. MacGowan, S. Mrowka, J. A. Koch, R. A. London, D. L. Matthews, and J. H. Underwood, "Power measurements of a saturated yttrium X-ray laser," Optics Lett., vol. 18, p. 1174, 1993.

[10] J. A. Koch, B. J. Mac Gowan, L. B. Da Silva, D. L. Matthews, J. H. Underwood, B. J. Batson, and S. Mrowka, "Observation of gain narrowing and saturation behavior in Se X-ray laser line profiles," Phys. Rev. Lett., vol. 68, p. 3291, 1992.

[11] B. Lemoff, G. Y. Yin, C. L. Gordon, III, C. P. J. Barty, and S. E. Harris, "Demonstration of a $10 \mathrm{~Hz}$, femtosecond pulse driven XUV laser at $41.8 \mathrm{~nm}$ in XeIX," Phys. Rev. Lett., vol. 74, p. 1574, 1995.

[12] J. J. Rocca, V. Shyaptsev, F. G. Tomasel, O. D. Cortazar, D. Hartshorn, and J. L. A. Chilla, "Demonstration of a discharge pumped table-top soft X-ray laser," Phys. Rev. Lett., vol. 73, p. 2192, 1994.

[13] J. J. Rocca, F. G. Tomasel, M. C. Marconi, V. N. Shlyaptsev, J. L. A. Chilla, B. T. Szapiro, and G. Giudice, "Discharge-pumped soft X-ray laser in neon-like argon," Phys. Plasmas, vol. 2, pp. 2547-2554, 1995.

[14] J. J. Rocca, O. D. Cortazar, B. Szapiro, K. Floyd, and F. G. Tomasel, "Fast discharge excitation of hot capillary plasmas for soft X-ray amplifiers," Phys. Rev. E, vol. 47, p. 1299, 1993.

[15] G. J. Linford, E. R. Peressini, W. R. Sooy, and M. L. Spaeth, "Very long lasers," Appl. Opt., vol. 13, p. 379, 1974.

[16] R. A. London, "Beam optics of exploding foil plasma X-ray lasers," Phys. Fluids, vol. 31, pp. 184-192, 1988.

[17] L. W. Casperson, "Threshold characteristics of mirrorless lasers," $J$. Appl. Phys., vol. 48, pp. 256-262, 1977.

[18] J. L. A. Chilla and J. J. Rocca, "Beam optics in gain guided soft X-ray lasers in cylindrical plasmas," to be published.

J. J. Rocca (S'80-M'83-SM'94), photograph and biography not available at the time of publication.

M. C. Marconi, photograph and biography not available at the time of publication.

J. L. A. Chilla, photograph and biography not available at the time of publication.

D. P. Clark, photograph and biography not available at the time of publication.

F. G. Tomasel, photograph and biography not available at the time of publication.

V. N. Shlyaptsev, photograph and biography not available at the time of publication. 